\title{
Molecular Gas and Star Formation in Void Galaxies
}

\author{
M. Das ${ }^{1}$, T. Saito ${ }^{2}$, D. Iono ${ }^{2}$, M. Honey ${ }^{1}$ and S. Ramya ${ }^{3}$ \\ ${ }^{1}$ Indian Institute of Astrophysics, Banaglore, India \\ email: mousumi@iiap.res.in \\ ${ }^{2}$ Department of Astronomy, The University of Tokyo, Tokyo 113-0033 \\ ${ }^{3}$ Shanghai Astronomical Observatory, Shanghai
}

\begin{abstract}
We present the detection of molecular gas using $\mathrm{CO}(1-0)$ line emission and followup $\mathrm{H} \alpha$ imaging observations of galaxies located in nearby voids. The $\mathrm{CO}(1-0)$ observations were done using the $45 \mathrm{~m}$ telescope of the Nobeyama Radio Observatory (NRO) and the optical observations were done using the Himalayan Chandra Telescope (HCT). Although void galaxies lie in the most underdense parts of our universe, a significant fraction of them are gas rich, spiral galaxies that show signatures of ongoing star formation. Not much is known about their cold gas content or star formation properties. In this study we searched for molecular gas in five void galaxies using the NRO. The galaxies were selected based on their relatively higher IRAS fluxes or $\mathrm{H} \alpha$ line luminosities. $\mathrm{CO}(1-0)$ emission was detected in four galaxies and the derived molecular gas masses lie between $(1-8) \times 10^{9} M_{\odot}$. The $\mathrm{H} \alpha$ imaging observations of three galaxies detected in $\mathrm{CO}$ emission indicates ongoing star formation and the derived star formation rates vary between from $0.2-1.0 \mathrm{M}_{\odot} y r^{-1}$, which is similar to that observed in local galaxies. Our study shows that although void galaxies reside in underdense regions, their disks may contain molecular gas and have star formation rates similar to galaxies in denser environments.
\end{abstract}

Keywords. ISM: molecules, galaxies: evolution, galaxies: ISM, cosmology: large-scale structure of universe.

\section{Introduction}

Voids contain a sparse but significant population of galaxies that are usually small, gas rich, late type galaxies (kreckel etal. 2012). The smaller voids are dominated by low surface brightness (LSB) dwarfs and irregular galaxies (karachentsev etal. 1999) but the larger voids also have a population of relatively bright galaxies that are often blue in color. These galaxies have ongoing star formation and are often interacting with companion galaxies in pairs or small groups along filaments in the voids (Beygu et al. 2013). Many questions remain regarding star formation in void environments; what is its nature - is it sporadic or continuous, what drives it and how is the star formation related to the location of the galaxies with respect to the filaments, walls and void interiors ?

One of the key elements for supporting star formation in galaxies is the presence of molecular hydrogen $\left(\mathrm{H}_{2}\right)$ gas. Although neutral hydrogen has been both detected and mapped in several voids, not much is known about the distribution of $\mathrm{H}_{2}$ gas in void galaxies. There have been two studies that have detected $\mathrm{CO}$ emission and estimated molecular gas masses in a total of five void galaxies (Sage et al. 1997; Beygu et al. 2013). These results indicate that the $H_{2}$ gas masses in void galaxies are comparable to those found in nearby star forming systems. In this study we searched for molecular gas in void galaxies to obtain a larger sample of such $H_{2}$ rich galaxies and carried out followup $\mathrm{H} \alpha$ imaging observations of some of the detected galaxies. Our main motivation was to understand how the cold gas masses relate to the star formation properties of these 

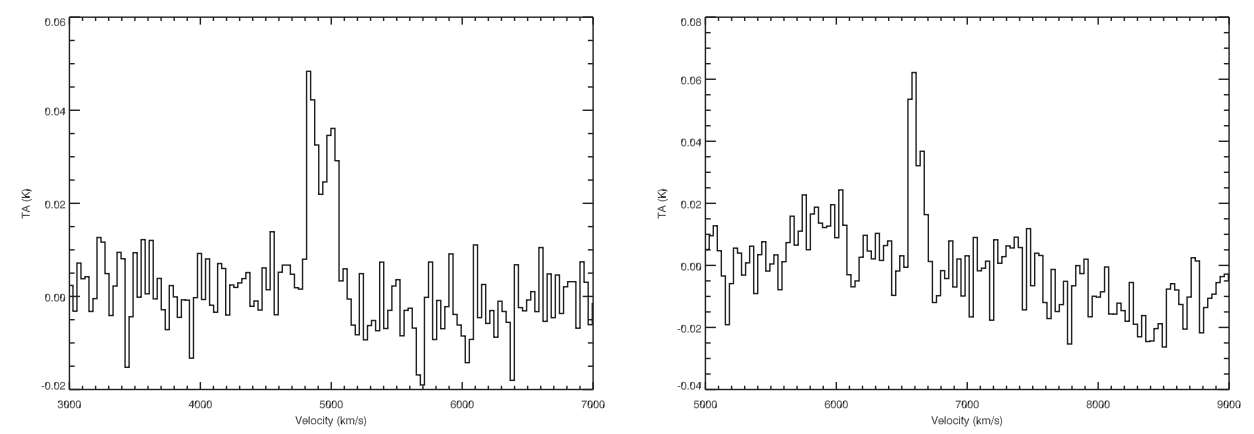

Figure 1. (a) Figure on the left shows the $\mathrm{CO}(1-0)$ line emission detected from the galaxy SBS $1325+597$ that lies in the void Ursa Minor I. The line has a distinctive double horned profile indicative of a rotating disk and the peak separation is approximately $200 \mathrm{~km} \mathrm{~s}^{-1}$. (b) Figure on the right shows the $\mathrm{CO}(1-0)$ line emission detected from the void galaxy SDSS $153821.22+331105.1$. The gas is concentrated in the center of the galaxy and has a line width of $\sim 100 \mathrm{~km} \mathrm{~s}^{-1}$.

systems. In the following sections we present our observations, results and discuss their implications. For all distances we have used $H_{0}=73 \mathrm{~km} \mathrm{~s}^{-1} \mathrm{Mpc}^{-1}$ and $\Omega=0.27$.

\section{Sample galaxies and observations}

Our initial sample comprised of 12 galaxies that were selected based of their relatively high infra-red fluxes or high star formation rates (kreckel et a. 2012; Cruzen et al. 2002; Szomoru et al. 1996). However due to weather conditions we were able to finally observe only five galaxies from this sample and they are listed in Table 1. Three galaxies have been observed in HI by Kreckel et al. (2012) in the Void Galaxy Survey (VGS) (SBS 1325+597, SDSS 143052.33+551440.0, SDSS 153821.22+331105.1) and the remaining two galaxies have been observed in an earlier HI survey of the Bootes void by Szomoru et al. (1996). All the galaxies have SDSS data.

The ${ }^{12} C O(J=1-0)$ emission observations were carried out using the $45 \mathrm{~m}$ Nobeyama Radio Telescope during 14 - 25 April, 2013. At the CO rest frequency of $115.271204 \mathrm{GHz}$, the half-power beam width (HPBW) was $15^{\prime \prime}$ and the main beam efficieny was about $30 \%$. The on source time for the first four galaxies varied between 1 to 1.5 hours; due to poor weather conditions SBS $1428+529$ was observed for only 25 minutes. We used the one beam (TZ1), dual polarization, double sideband receiver (TZ) and the digital FX-type spectrometer SAM45, that has a bandwidth of $4 \mathrm{GHz}$ (Nakajima et al. 2008). Typical system temperatures were $160-260 \mathrm{~K}$. The pointing accuracy was about $2^{\prime \prime}-4^{\prime \prime}$. Only data with a wind velocity less than $5 \mathrm{~km} \mathrm{~s}^{-1}$ were used and data with winding baselines were flagged. The data was analysed using NRO calibration tool NEWSTAR.

The $\mathrm{H} \alpha$ observations were done using the Himalayan Faint Object Spectrograph Camera (HFOSC) which is mounted on the $2 \mathrm{~m}$ Himalayan Chandra Telescope (HCT) and were carried out on 2014 April $11 \& 25$. For SBS $1325+597$ the redshift is 0.0165 , so the $\mathrm{H} \alpha$ filter (band width $\sim 500 \AA$ ) was used to get the $\mathrm{H} \alpha$ line emission. For SDSS $143052.33+551440.0$ we used the narrow $\mathrm{H} \alpha$ filter (band width $\sim 100 \AA$ ). The galaxy SDSS J153821.22+331105.1 is at a redshift of $z=0.022$ and the $\mathrm{H} \alpha$ line is shifted to $6714 \AA$. Hence we used the narrow band $[\mathrm{SII}]$ filter (band width $\sim 100 \AA$ and centered around $6724 \AA$ ) for this galaxy. To obtain the continuum subtracted $\mathrm{H} \alpha$ images we also obtained broad band images with the $R$ filter centered around the $\mathrm{H} \alpha$ line. The bias frames and twilight flats were used for preprocessing of the images. The data reduction 
Table 1. Observed galaxies, molecular gas masses and star formation rates

\begin{tabular}{|c|c|c|c|c|c|c|}
\hline Galaxy & $\begin{array}{c}\mathbf{D}_{L} \\
\mathbf{M} \mathbf{p c}\end{array}$ & Redshift & $\begin{array}{c}\text { CO flux } \\
\left(\mathrm{K} \mathrm{km} \mathrm{s}^{-1}\right)\end{array}$ & $\begin{array}{c}\text { Molecular Gas } \\
\text { Mass }\left(\begin{array}{ll}10^{9} & M_{\odot}\end{array}\right)\end{array}$ & $\begin{array}{l}\mathrm{H} \alpha \text { Flux }\left(10^{-13}\right. \\
\left.\operatorname{ergs~s}^{-1} \mathrm{~cm}^{-2}\right)\end{array}$ & $\begin{array}{c}\mathbf{S F R} \\
\left(M_{\odot} y r^{-1}\right)\end{array}$ \\
\hline SBS $1325+597$ & 70.4 & 0.0165 & $10.7 \pm 0.2$ & $1.5 \pm 0.03$ & 0.4 & 0.20 \\
\hline SDSS $143052.33+551440.0$ & 76.6 & 0.0176 & $7.0 \pm 0.2$ & $1.1 \pm 0.03$ & 1.2 & 0.60 \\
\hline SDSS $153821.22+331105.1$ & 97.6 & 0.0220 & $6.4 \pm 0.2$ & $1.7 \pm 0.05$ & 1.2 & 1.02 \\
\hline CG 598 & 248.0 & 0.0575 & $5.2 \pm 0.1$ & $8.5 \pm 0.10$ & $\ldots$ & $\ldots \ldots$ \\
\hline SBS $1428+529$ & 191.0 & 0.0445 & $<0.6$ & $<0.6$ & $\ldots$ & ..... \\
\hline
\end{tabular}

Notes:

1 For SBS $1428+529$ there was no $\mathrm{CO}(1-0)$ detection and no $\mathrm{H} \alpha$ image could be obtained. Upper limits for the molecular gas mass were obtained from the noise which was $0.0024 \mathrm{k}$ and assuming a typical linewidth of $250 \mathrm{~km} / \mathrm{s}$.

${ }^{2}$ For $\mathrm{CG} 598$ no $\mathrm{H} \alpha$ image could be obtained.

was done using the standard packages available in IRAF $\dagger$. The images were corrected for cosmic rays, aligned and corrected for point spread function variations. Flux calibration was done using the spectrophotometric standard star HZ44. The $\mathrm{H} \alpha$ fluxes are listed in Table 1.

\section{Results}

1. Molecular gas detection : We have detected ${ }^{12} C O(J=1-0)$ emission from four of the five sample galaxies that we observed (Table 1). The non-detection in SBS 1428+529 could partly be due to the short duration of the scan, which was limited by bad weather. Of the four detections, SBS $1325+597$ has the most striking line profile; it has a double horned structure indicating a rotating disk of molecular gas (Figure 1a). The velocity separation of the peaks is $\sim 200 \mathrm{kms}^{-1}$; assuming a disk inclination of $59.3^{\circ}$ the disk rotation is $116 \mathrm{~km} \mathrm{~s}^{-1}$. This is similar to the HI rotation speed from Kreckel et al. (2012). In SDSS $153821.22+331105.1$ the gas is centrally peaked (Figure 1b); probably driven into the center by the bar in the galaxy. In the other two galaxies (SDSS 143052.33+551440.0 and CG 598) the CO line profile is slightly off center from the systemic velocities of the galaxies, which suggests that their gas disks are disturbed, possibly due to interaction with a companion galaxy (Das et al. 2014, in preparation).

2. Molecular masses : The CO fluxes in $\mathrm{K} \mathrm{km} \mathrm{s}^{-1}$ were converted to $\mathrm{Jy} \mathrm{km} / \mathrm{s}$ using a conversion factor $(\mathrm{Jy} / \mathrm{K})$ of 2.4. The $\mathrm{CO}$ line luminosity was determined using the relation $L_{C O}=3.25 \times 10^{7}\left(S_{C O} \Delta V / J_{y k m s}{ }^{-1}\right)\left(D_{L} / M p c\right)^{2}\left(\nu_{r e s}\right)^{-2}(1+z)^{-1}$ and the molecular gas mass was estimated using the relation $M\left(H_{2}\right)=\left[4.8 L_{C O}\left(K_{k m s^{-1}}\right)\right]$ (Solomon \& van den Bout 2005). The molecular gas masses lie in the range $(1-8) \times 10^{9} M_{\odot}$ which is comparable to that observed from bright galaxies in denser environments.

3. Comparison with previous detections and HI masses : Our molecular gas are similar to that obtained for earlier studies of void galaxies by (Sage et al. 1997, Beygu et al. 2013) that lie in the range $10^{8}-10^{9} \mathrm{M}_{\odot}$. Although our study and previous detections indicate relatively large $H_{2}$ gas masses and a high detection rate, it must be remembered that the sample was biased towards star forming galaxies and those with high FIR fluxes. The molecular gas masses are comparable to the HI masses of these galaxies (Kreckel et al. 2012; Szomoru et al. 1996).

4. $\mathbf{H} \alpha$ fluxes and star formation rates (SFR) : The SFRs were calculated from the $\mathrm{H} \alpha$ fluxes using the kennicutt formula $\mathrm{SFR}=\mathrm{L}(\mathrm{H} \alpha) / 1.26 \times 10^{41} \operatorname{ergs~s}^{-1}$ (Table 1).

$\dagger$ Image Reduction \& Analysis Facility Software distributed by National Optical Astronomy Observatories, which are operated by the Association of Universities for Research in Astronomy, Inc., under co-operative agreement with the National Science Foundation. 
In SBS $1325+597$ the $\mathrm{H} \alpha$ is distributed on either side of the galaxy nucleus, possibly in a ring. The distrbution matches the CO line profile (Figure 1a) which indicates a ring like configuration for the molecular gas. In SDSS 143052.33+551440.0 the $\mathrm{H} \alpha$ emission is concentrated about the nucleus. In SDSS 153821.22+331105.1 it is concentrated along the bar. The SFR is highest for SDSS $153821.22+331105.1$; it is probably triggered by gas flowing along the bar in the galaxy although the emission is surprisingly faint in the disk.

5. Are these galaxies interacting? : SBS $1325+597$ has a disturbed optical and HI morphology. SDSS $143052.33+551440.0$ also has a disturbed optical morphology and its CO profile is not symmetric about its systemic velocity. SDSS $153821.22+331105.1$ has a bar that may have been triggered by an interaction. CG 598 appears to be accreting a companion in its SDSS g image and its CO profile is also asymmetric about its systemic velocity. Thus all the detected galaxies in our sample show some signs of interaction.

\section{Implications}

The main implications of this study is that cold gas and star formation are present in voids, even though the overall environment is underdense. Our sample galaxies also show disturbed morphologies, possibly due to interactions with companion galaxies. Our results can be understood in the hierarchical picture of void evolution, in which voids merge leaving behind a filamentary substructure. Galaxies grow along these filaments and in clusters where filaments intersect (e.g. Sahni et al. 1994; Sheth \& van de Weygaert 2004; Cautun et al. 2014). The presence of both molecular gas and star formation in void galaxies indicates that they are probably evolving within this void substructure. Gas flowing along the filaments can be accreted by these galaxies and will contribute to the accumulation of neutral gas in their disks. High enough gas surface densities will result in the onset of star formation, leading to galaxy evolution within the void environment.

\section{Acknowledgements}

This paper was based on observations at the Nobeyama Radio Observatory (NRO) which is a branch of the National Astronomical Observatory of Japan, National Institutes of Natural Sciences. The optical observations were done at the Indian Optical Observatory (IAO) at Hanle. We thank the staff of IAO, Hanle and CREST, Hosakote, that made these obervations possible. This research has made use of the NASA/IPAC Extragalactic Database (NED).

\section{References}

Beygu, B., Kreckel, K., van de Weygaert, R., et al. 2013, AJ, 145, 120

Cautun, M., van de Weygaert, R., Jones, B. J. T. et al. 2014, MNRAS, 441, 2923

Cruzen, Shawn, Wehr, Tara, Weistrop, Donna et al. 2002, AJ, 123, 142

Karachentsev, V. E., Karachentsev, I. D., \& Richter, G. M. 1999 AESAS, 135, 221

Kreckel, K., Platen, E., Aragn-Calvo, M. A., et al. 2012, AJ, 144, 16

Kreckel, K., Platen, E., Aragn-Calvo, M. A., et al. 2011, AJ, 141, 4

Nakajima, Taku, Sakai, Takeshi, Asayama, Shin'ichiro, et al. 2008, PASJ, 60, 435

Sage, L. J., Weistrop, D., Cruzen, S., \& Kompe, C. 1997, AJ, 114, 1753

Sahni, Varun, Sathyaprakah, B. S., Shandarin, \& Sergei F. 1994, ApJ, 431, 20

Sheth, Ravi K. \& van de Weygaert, Rien 2004, MNRAS, 350, 517

Solomon, P. M. \& Vanden Bout, P. A. 2005, ARA\&A, 43, 677

Szomoru, Arpad, van Gorkom, J. H., Gregg, \& Michael D. 1996, AJ, 111, 2141 